\title{
Trichoderma harzianum UN BIOCONTROL Y BIOPROMOTOR EN VIVERO DE ESPECIES FORESTALES
}

\author{
Graciela Romero, Alicia Crosara y Amalia Baraibar'
}

\section{RESUMEN}

El uso de fungicidas para controlar problemas sanitarios en viveros forestales presenta problemas de manejo tanto por su toxicidad como por los problemas de resistencia de los patógenos. Esto lleva a incrementar la cantidad de productos aplicados o la frecuencia de las aplicaciones sin lograr en ciertos casos el efecto de control deseado.

En los viveros forestales son frecuentes ataques por diferentes patógenos, según se trate de eucaliptos o de pinos. En el caso de patógenos que atacan el genero Pinus, infecciones de Botrytis cinerea Pers ex Pers, agente causal del moho gris de los pinos; Fusarium spp, agente causal de Damping-off, se manifiestan en el transcurso de la producción de plantas. Trichoderma harzianum es un agente de biocontrol de enfermedades y promotor de crecimiento que tiene potencial aplicación en viveros forestales.

El objetivo de este trabajo es lograr la inclusión de Trichoderma en el sistema sustrato - plantin forestal y los resultados productivos, a travès de la selección de sustratos y el ajuste de los esquemas nutricionales empleados en un vivero comercial productor de plantas de pino y eucalipto.

Se estudió el comportamiento de una cepa de Trichoderma harzianum (Trichosoil, Lage y Cia SA) en dos sustratos de uso comercial. Se evaluó la supervivencia del antagonista durante el ciclo de producción de plantines de Eucalyptus grandis, E. globulus y Pinus taeda. Los sustratos esterilizados fueron inoculados con Trichosoil $\left(3 \mathrm{~kg} / \mathrm{m}^{3}\right)$, incubados por 3 dias y distribuidos en bandejas posteriormente sembradas con las diferentes especies. Periódicamente se efectuó recuentos de ufc./g en cada sustrato. Para cada fase de crecimiento de los plantines (arranque, cria y terminación) se efectuó los tratamientos nutricionales según el esquema de la Empresa Colonvade SA, manteniendo la relación entre nutrientes y variando la dosis al doble y al cuádruple.

Se constató diferencias significativas entre sustratos en el crecimiento y supervivencia de Trichoderma. El sustrato en base a cáscara de pino compostada mantuvo las poblaciones inicialmente incorporadas $(6 \times 105 \mathrm{ufc} / \mathrm{g})$ hasta el final del periodo del ensayo.

Se determinó también parámetros de calidad de los plantines obtenidos como peso

1 ingeniera Agrónoma, Prolesora Prolección Forestal Departamento Forestal, Facultad de Agronomia labgrom@adinetcom.uy Ingeniera Agronoma M Sc. Facultad Ciencias UNCIEP crosara@fcien.edu.uy

Ingeniera Agrónoma M Sc. Lage \& Cia. S A. lage@lageycia com.uy 
seco y fresco de raiz y parte aérea, diámetro de cuello y altura de las plantas obtenidos en los diferentes tratamientos realizados.

Se constató efecto promocional de Trichoderma en niveles de $12 \%$ a $50 \%$ en parámetros de altura, diámetro, peso radicular y de la parte aérea, entre otros, por encima de los valores obtenidos con la tecnología convencional.

Estos resultados son alentadores en aspectos productivos y económicos, ya que con la inclusión de Trichosoil y el ajuste nutricional correspondiente, se logró uniformar la calidad de los plantines a la vez que acortar el periodo de preparación de plantas, liberando al vivero para futuras nuevas plantaciones

\section{SUMMARY}

Fungicides used to control forest diseases in nurseries often show problems due to toxicity produced in its management and also due to resistence in pathogens. These leads to increase either the dosage or the application frequency, in most cases without getting the disease control.

Diseases in forest nurseries are usually produced by pathogens like Botrytis cinerea, Fusarium spp on Pines and Eucalypts seedlings.

The objectives of this paper were to include the use o Trichoderma in the management practice of a commercial nursery producing Pines and Eucalypts seedlings. Another objective was the nutritional evaluation of the produced seedlings as well as the quality parameters for seedling production related to the effect of the biocontrol agent.

The test included the study of Trichoderma harzianum in two different commercial substrates to evaluate surviving conditions of the bioagent of Pinus taeda, Eucalyptus grandis and $E$. globulus seddlings. The substrate was initially sterilized and inoculated with Trichosoil (3 $\left.\mathrm{k} / \mathrm{m}^{3}\right)$, incubated during 3 days and sawn with seeds of the different species. Countings of units of forming colony numbers were collected periodically for each substrate.

In each seedling growing stage (starting, growing and termination) nutritional treatments were followed as the nursery carried out commonly keeping the relation for nutrients and also making double and quadruple dosage.

Significant differences were detected on Trichoderma grow and survival in substrates.

The composted pine cork substrate gave higher survival number for cfu (colony forming units) and kept initial population of Trichoderma cfu up to the end of the test.

Quality parameters for seedling production were also evaluated as fresh and dried weight for root and aerial components. 


\section{MATERIALES Y METODOS}

\section{Colonización y Supervivencia de Trichoderma harzianum en Dos Sustratos Comerciales}

\section{- En Ausencia de Plantas}

Previo a la instalación del ensayo en el vivero, se incorporó el agente microbiano Trichoderma harzianum cepa L1, producido comercialmente por la firma Lage y Cia con el nombre de Trichosoil, a los dos sustratos comerciales. La dosis empleada fue de $3 \mathrm{~kg} / \mathrm{m}^{3}$ de cada sustrato, que representa una población esperada de conidios o propágulos equivalente a $1.5 \times 10^{6}$ ufc por celda de las bandejas. Se mezcló bien el producto en cada sustrato y se dejó incubando a temperatura ambiente para promover la colonización. Se llenó las bandejas con los dos sustratos inoculados y los testigos respectivos sin inocular. Fueron 2 sustratos $x 2$ tratamientos de inoculación $\times 5$ fechas de recuentos $\times 3$ repeticiones. Cada repetición (parcela) estaba constituida por una bandeja con 72 celdas. Para este estudio no se incorporó semillas.

Se midió la colonización y supervivencia del Trichoderma inoculado en los dos sustratos mediante el recuento de las ufc./g en medio selectivo, a partir de muestras de $10 \mathrm{~g}$ de cada sustrato, provenientes de una mezcla de diferentes celdas al azar de cada repetición y por el método de dilución en placas, con tres repeticiones por tratamiento. Se contabilizó las ufc./g a las $0,1,3,9,18$ y 23 semanas del experimento.

\section{En Presencia de Plantas}

En base al esquema de manejo de los sustratos, se instaló un experimento con plantas considerando Pinus taeda, Eucalyptus grandis y E.globulus, lo que contabiliza 12 tratamientos ( 3 especies $\times 2$ sustratos $\times 2$ tratamientos al sustrato) con 6 repeticiones ( 1 bandeja con 72 plantas $=1$ repetición). Se evaluó parámetros morfológicos en los plantines (altura y peso de la materia seca) a las 13,18 y 23 semanas. Solamente se contabilizó las ufc./g al comienzo del experimento y al cosechar, siguiendo la misma metodologia.

\section{Evaluación de Diferentes Dosis de Fertilización en la Producción de Plantines de Pinus taeda Creciendo en Sustrato Colonizado por Trichoderma harzianum}

El experimento se instaló en un invernáculo del vivero "La Buena Unión" de la empresa COLONVADE S.A. ubicado en el departamento de Rivera, Uruguay. Se utilizó 80 bandejas de PVC negro de 72 celdas cada una con una capacidad de $93 \mathrm{~cm}^{3}$ por celda.

Se empleó el sustrato de la empresa cuyo componente principal es cáscara de pino compostada. La inoculación del sustrato se efectuó de la misma forma arriba detallada y se realizó el recuento a los 3 meses de la inoculación.

Se aplicó 5 tratamientos con 4 repeticiones por tratamiento. La diferencia de tratamientos corresponde a diferentes dosis de fertilización, con Trichoderma en el sustrato (T1, T2, T3 y T4) y un testigo sin Trichoderma (TO) y con el nivel de fertilización de T1. El detalle es presentado 
en el Cuadro $\mathrm{N}^{\circ} 1$.

La fertilización se realizó via foliar en todas sus etapas y con frecuencia semanal.

El diseño estadistico fue de bloques completos al azar con 4 bloques, 5 tratamientos y 4 repeticiones por tratamiento.

Los parámetros morfológicos evaluados fueron altura y diámetro de cuello y los parámetros fisiológicos peso fresco y seco de plantines y dosis de macro y micro nutrientes (en la semana 16 y 21 ).

En ningún momento del ensayo se aplica fungicidas.

Cuadro $\mathrm{N}^{\circ} 1$

DESCRIPCIÓN DE TRATAMIENTOS

\begin{tabular}{|c|c|c|c|c|c|c|}
\hline Etapa & Fertilizante & $\begin{array}{c}\text { T0 } \\
\text { (g/planta) }\end{array}$ & $\begin{array}{c}\text { T 1 } \\
\text { (g/planta) }\end{array}$ & $\begin{array}{c}\text { T } 2 \\
\text { (g/planta) }\end{array}$ & $\begin{array}{c}\text { T } 3 \\
\text { (g/planta) }\end{array}$ & $\begin{array}{c}\text { T4 } \\
\text { (g/planta) }\end{array}$ \\
\hline Arranque & $\begin{array}{l}18-44-0 \\
13-0-46\end{array}$ & $\begin{array}{l}0.010 \\
0.006\end{array}$ & $\begin{array}{l}0.010 \\
0.006\end{array}$ & $\begin{array}{l}0.005 \\
0.003\end{array}$ & $\begin{array}{l}0.020 \\
0.012\end{array}$ & $\begin{array}{l}0.040 \\
0.024\end{array}$ \\
\hline Cria & $\begin{array}{l}18-18-18 \\
34-0-0 \\
18-18-18 \\
13-0-46 \\
15-5-0+\mathrm{Ca}\end{array}$ & $\begin{array}{r}0.015 \\
0.004 \\
\\
0.015 \\
0.0035 \\
0.0035\end{array}$ & $\begin{array}{r}0.015 \\
0.004 \\
\\
0.015 \\
0.0035 \\
0.0035\end{array}$ & $\begin{array}{r}0.0075 \\
0.002 \\
\\
0.0075 \\
0.00175 \\
0.00175\end{array}$ & $\begin{array}{r}0.03 \\
0.008 \\
\\
0.03 \\
0.007 \\
0.007\end{array}$ & $\begin{array}{r}0.06 \\
0.016 \\
\\
0.06 \\
0.014 \\
0.014\end{array}$ \\
\hline Terminación & $\begin{array}{l}11-9-35 \\
0-52-34\end{array}$ & $\begin{array}{l}0.013 \\
0.009\end{array}$ & $\begin{array}{l}0.013 \\
0.009\end{array}$ & $\begin{array}{l}0.0065 \\
0.0045\end{array}$ & $\begin{array}{l}0.026 \\
0.018\end{array}$ & $\begin{array}{l}0.052 \\
0.036\end{array}$ \\
\hline
\end{tabular}

T 0

$\mathrm{T} 1-\mathrm{T} 2-\mathrm{T} 3-\mathrm{T}-4$

Sin Trichoderma harzianum

Con Trichoderma harzianum

\section{RESULTADOS}

\section{Colonización y Supervivencia de Trichoderma harzianum en Dos Sustratos Comerciales}

\section{- En Ausencia de Plantas}

Se observó diferencias estadisticamente significativas entre sustratos, siendo el Sustrato 1 (cáscara de pino compostado) el que presentó más altos recuentos de Trichoderma. Al cabo de las 23 semanas, no se detectó descensos poblacionales en este sustrato lo que indica su capacidad para mantener viable al agente microbiano. A partir de la novena semana, se constató la presencia de una cepa de Trichoderma nativa del sustrato, que se desarrolló en las sucesivas semanas llegando a niveles similares al Trichoderma inoculado. Sus caracteristicas 
culturales son bien diferentes de la cepa $\mathrm{L} 1$.

\section{Cuadro $\mathrm{N}^{\mathrm{a}} 2$}

\section{COLONIZACIÓN Y SOBREVIVENCIA DE Trichoderma harzianum EN DOS SUSTRATOS COMERCIALES PARA CULTIVO DE ESPECIES FORESTALES EN VIVERO}

\begin{tabular}{|c|c|c|c|c|c|}
\hline \multirow{3}{*}{ Tratamientos } & \multicolumn{5}{|c|}{ Trichoderma (ufcig de sustrato) } \\
\hline & \multicolumn{5}{|c|}{ Tiempo (semanas) } \\
\hline & 0 & $1,3^{*}$ & 9 & 18 & 23 \\
\hline Sustrato $1\left(\mathrm{~S}_{1}\right)$ & nsd & nsd & $5,83 \times 10^{4} \mathrm{~b}$ & $1,07 \times 10^{5} \mathrm{ab}$ & $2.67 \times 10^{5} \mathrm{a}$ \\
\hline $\mathrm{S}+\mathrm{T}$. harzianum & $5,6 \times 10^{5}$ & $1 \times 10^{6} \mathrm{a}$ & $3,07 \times 10^{5} \mathrm{a}$ & $2,43 \times 10^{5} \mathrm{a}$ & $3,3 \times 10^{5} \mathrm{a}$ \\
\hline Sustrato $2\left(\mathrm{~S}_{2}\right)$ & nsd & nsd & nsd & $1,5 \times 10^{3} \mathrm{~b}$ & $2,3 \times 10^{3} \mathrm{~b}$ \\
\hline \multirow[t]{3}{*}{$\mathrm{S}_{\mathrm{f}}+\mathrm{T}$. harzianum } & $4,5 \times 10^{5}$ & $4,5 \times 10^{5} \mathrm{~b}$ & $1.6 \times 10^{4} \mathrm{~b}$ & $3,13 \times 10^{4} \mathrm{~b}$ & $3,43 \times 10^{4} b$ \\
\hline & F: sd & F: sd & F: sd & $F: 0,82$ & $F: 0,09$ \\
\hline & DMS: 0,0 & DMS: 0,0 & DMS: 0,0 & $\begin{array}{c}\text { DMS: } \\
1,57 \times 10^{5}\end{array}$ & $\begin{array}{c}\text { DMS: } \\
1,32 \times 10^{=}\end{array}$ \\
\hline
\end{tabular}

nsd: no se detectó colonias de Trichoderma en la menor dilución empleada para el recuento.

- siembra del ensayo

A partir de la $16^{\text {a }}$ semana se comenzó con el plan de fertilización via riego.

Letras distintas indican diferencias significativas $(p<=0,1)$.

En cuanto al sustrato 2, este no fue capaz de sostener los niveles de inoculo agregados al inicio. Las diferencias principales por las que estos dos sustratos se contraponen tienen relación con la naturaleza de la fracción orgánica que los compone. En el sustrato 1, por ser un compost, la fracción de $\mathrm{C}$ es más lábil que en el sustrato 2, compuesta fundamentalmente por ligninas estabilizadas. Trichoderma por ser un hongo celulolitico, germinó preferentemente en el sustrato con fuentes de Carbono más mineralizables que están presentes en el Sustrato 1. Esto concuerda con Steiner y Lockwood (1969), Brodie y Blakeman (1976) y, más recientemente, Nelson, Kuter y Hoitnik (1983). Se demostró la importancia de la elección del sustrato y las caracteristicas del mismo cuando se introduce un agente microbiano, que deberá sobrevivir y mantenerse viable para ejercer su antagonismo o actividad de promoción de crecimiento. La dinámica de la colonización y supervivencia concuerda con las aseveraciones de Papavizas (1985).

\section{- En Presencia de Plantas}

Las mediciones de altura de plantin de las 3 especies a las 13 semanas son indicadoras del efecto inmovilizador de la presencia de Trichoderma en los sustratos. Para el caso del Sustrato 2 la falta de nutrientes disponibles para la planta se observó aun en los tratamientos sin el Trichoderma. La estabilidad de los componentes del Sustrato 2, muy utilizado en producciones horticolas, pero con fertilización complementaria a partir de los 7 dias, explica la falta de respuesta en el crecimiento. 


\section{Cuadro $\mathrm{N}^{\circ} 3$ \\ EVOLUCIÓN DE LA ALTURA DE PLANTINES FORESTALES DE Pinus taeda QUE CRECEN EN DOS SUSTRATOS COLONIZADOS O NO CON Trichoderma harzianum.}

\begin{tabular}{|l|c|c|c|}
\hline \multirow{2}{*}{ Tratamiento } & \multicolumn{3}{|c|}{ Altura de plantin (cm) } \\
\cline { 2 - 4 } & 13 & 18 & 23 \\
\cline { 2 - 4 } Sustrato 1 $\left(\mathrm{S}_{1}\right)$ & $9,65 \mathrm{a}$ & $12,17 \mathrm{a}$ & $13,53 \mathrm{a}$ \\
\hline $\mathrm{S}_{1}+$ T. harzianum & $7,78 \mathrm{~b}$ & $9,68 \mathrm{~b}$ & $11,33 \mathrm{~b}$ \\
\hline Sustrato 2 $\left(\mathrm{S}_{2}\right)$ & $8,52 \mathrm{~b}$ & $10,20 \mathrm{~b}$ & $11,00 \mathrm{~b}$ \\
\hline $\mathrm{S}_{2}+$ T. harzianum & $8,43 \mathrm{~b}$ & $9,82 \mathrm{~b}$ & $10,58 \mathrm{~b}$ \\
\hline \multirow{2}{*}{} & $\mathrm{F}: 4,86$ & $F: 11,93$ & $\mathrm{~F}: 6,3$ \\
\cline { 2 - 4 } & DMS: 0,987 & DMS: 0,742 & DMS: 0,866 \\
\cline { 2 - 4 } & & & \multicolumn{3}{|c|}{ Tiemponas) } \\
\hline
\end{tabular}

*A partir de la semana 16 se comenzó con el plan de fertilización via riego.

Letras distintas indican diferencias significativas $(p<=0.1)$

\section{Cuadro $\mathrm{N}^{\mathrm{a}} 4$}

EVOLUCIÓN DE LA ALTURA DE PLANTINES FORESTALES DE Eucalyptus globulus QUE CRECEN EN DOS SUSTRATOS COLONIZADOS O NO CON Trichoderma harzianum.

\begin{tabular}{|l|c|c|c|}
\hline \multirow{2}{*}{ Tratamiento } & \multicolumn{3}{|c|}{ Altura de Plantín (cm) } \\
\cline { 2 - 4 } & 13 & 18 & 23 \\
\hline Sustrato 1 $\left(\mathrm{S}_{1}\right)$ & $18,63 \mathrm{a}$ & $22,70 \mathrm{ab}$ & $25,35 \mathrm{c}$ \\
\hline $\mathrm{S}_{1}+\mathrm{T}$. harzianum & $15,92 \mathrm{ab}$ & $21,23 \mathrm{~b}$ & $25,62 \mathrm{bc}$ \\
\hline Sustrato 2 $\left(\mathrm{S}_{2}\right)$ & $14,95 \mathrm{~b}$ & $23,33 \mathrm{ab}$ & $29,86 \mathrm{ab}$ \\
\hline $\mathrm{S}_{2}+$ T. harzianum & $16,18 \mathrm{ab}$ & $25,35 \mathrm{a}$ & $31,45 \mathrm{a}$ \\
\hline & $\mathrm{F}: 2,78$ & $\mathrm{~F}: 2,29$ & $\mathrm{~F}: 0,13$ \\
\hline & DMS: 2,888 & DMS: 2,806 & DMS: 4,503 \\
\hline
\end{tabular}

${ }^{*}$ A partir de la $16^{\mathrm{a}}$ semana se comenzó con el plan de fertilización via riego.

Letras distintas indican diferencias significativas ( $p<=0.1$ )

Solamente para el caso de Eucalyptus globulus se observó compensación a partir del inicio del plan de fertilizaciones (13 semanas). 


\section{Cuadro $\mathrm{N}^{\circ} 5$ \\ EVOLUCIÓN DE LA ALTURA DE PLANTINES FORESTALES DE Eucalyptus grandis QUE CRECEN EN DOS SUSTRATOS COLONIZADOS O NO CON Trichoderma harzianum.}

\begin{tabular}{|l|c|c|c|}
\hline \multirow{2}{*}{ Tratamiento } & \multicolumn{3}{|c|}{ Altura de plantin (cm) } \\
\cline { 2 - 4 } & 13 & 18 & 23 \\
\hline Sustrato 1 $\left(\mathrm{S}_{1}\right)$ & $25,58 \mathrm{a}$ & $30,83 \mathrm{a}$ & $33,88 \mathrm{a}$ \\
\hline $\mathrm{S}_{1}+\mathrm{T}$. harzianum & $22,25 \mathrm{~b}$ & $27,38 \mathrm{~b}$ & $30,98 \mathrm{ab}$ \\
\hline Sustrato 2 $\left(\mathrm{S}_{2}\right)$ & $11,38 \mathrm{c}$ & $22,08 \mathrm{c}$ & $27,02 \mathrm{bc}$ \\
\hline $\mathrm{S}_{2}+\mathrm{T}$. harzianum & $11,55 \mathrm{c}$ & $21,22 \mathrm{c}$ & $26,52 \mathrm{c}$ \\
\hline & $\mathrm{F}: 2,52$ & $\mathrm{~F}: 1,19$ & $\mathrm{~F}: 0,47$ \\
\hline & DMS: 2,653 & DMS: 2,892 & DMS: 4,269 \\
\hline
\end{tabular}

* A partir de la $16^{\text {a }}$ semana se comenzó con el plan de fertilización vía riego.

Letras distintas indican diferencias significativas $(p<=0.1)$

\section{Cuadro $\mathrm{N}^{\circ} 6$}

EVOLUCIÓN DEL PESO DE LA MATERIA SECA DE PLANTINES FORESTALES CRECIENDO EN DOS SUSTRATOS COLONIZADOS O NO CON Trichoderma harzianum.

\begin{tabular}{|c|c|c|c|c|c|c|c|c|c|}
\hline \multirow{4}{*}{ Tratamiento } & \multicolumn{9}{|c|}{ Peso Materia Seca (g) } \\
\hline & \multicolumn{3}{|c|}{ P. taeda } & \multicolumn{3}{|c|}{ E. globulus } & \multicolumn{3}{|c|}{ E. grandis } \\
\hline & \multicolumn{9}{|c|}{ Tiempo (semanas) } \\
\hline & 13 & 18 & 23 & 13 & 18 & 23 & 13 & 18 & 23 \\
\hline Sustrato $1\left(\mathrm{~S}_{\mathrm{f}}\right)$ & 8,04 & 9,15 & 11,72 & 12,76 & 14,3 & 18,82 & 17,97 & 19,64 & 29,46 \\
\hline$S_{1}+T$. harzianum & 5,05 & 5,09 & 7,66 & 8,39 & 14,17 & 16,19 & 17,78 & 20,26 & 24,38 \\
\hline Sustrato $2\left(\mathrm{~S}_{2}\right)$ & 4,03 & 4,6 & 6.33 & 5,73 & 8,33 & 18,13 & 4,77 & 8,13 & 15,49 \\
\hline $\mathrm{S}_{2}+\mathrm{T}$. harzianum & 4,09 & 5,46 & 7,43 & 6,59 & 10,00 & 17,08 & 4,91 & 8,52 & 14,15 \\
\hline
\end{tabular}

Estos valores refieren al peso de 30 plantines.

* A partir de la $16^{\text {a }}$ semana se comenzó con el plan de fertilización via riego.

\section{Pinus taeda}

Los plantines de $\mathrm{S}_{1}$ fueron aproximadamente $100 \%$ más pesados que los de $\mathrm{S}_{2}$ a lo largo de todo el ciclo. Hubo respuesta importante a la fertilización a lo largo del ciclo, siendo el incremento del $14 \%$ de 13 a 18 semanas y de $28 \%$ de 18 a 23 semanas para el $\mathrm{S}_{1}$. Para el $\mathrm{S}_{2}$, dichos incrementos fueron de $14 \%$ y $38 \%$, respectivamente, en los dos momentos.

La presencia de Trichoderma no fue positiva en S, pues se redujeron los valores de materia seca en $37 \%$ a las 13 semanas, $44 \%$ a las 18 y $35 \%$ a las 23 semanas. En $S_{2}$, 
luego de la fertilización se observó incrementos de $19 \%$ y $17 \%$ a las 18 y 23 semanas, respectivamente, cuando se inoculaba el sustrato con Trichoderma.

\section{Eucalyptus globulus}

Los plantines creciendo en $\mathrm{S}_{1}$ a las 13 semanas tuvieron $123 \%$ más peso de la materia seca que los correspondientes al $\mathrm{S}_{2}$. Sin embargo, en las sucesivas mediciones esta diferencia fue menor alcanzando a las 18 semanas el $42 \%$ y desapareciendo a las 23 semanas.

Luego de la fertilización a la semana 16 , se produjo un incremento del $12 \%$ hasta la semana 18 y de $32 \%$ hasta la semana 23 en el $S_{1}$; en el $S_{2}$ los valores fueron de $45 \%$ y $118 \%$, respectivamente.

El agregado de Trichoderma promovió una reducción de la materia seca de los plantines creciendo en $\mathrm{S}_{1}$, tal como fue observado para el peso de la materia fresca. Esta reducción fue de $34 \%$ aproximadamente a las 13 semanas y $14 \%$ a las 23 semanas. La incorporación de Trichoderma en $\mathrm{S}_{2}$ fue positiva antes y después de la fertilización.

\section{Eucalyptus grandis}

Se observó diferencias muy importantes a favor del $S_{1}$ en el desarrollo de los plantines. Este sustrato fue $277 \%$ superior al $\mathrm{S}_{2}$ antes de incorporar el fertilizante. Luego de las 16 semanas, la ventaja de $S_{1}$ sobre $S_{2}$ fue de $142 \%$ a las 18 semanas y de $47 \%$ a las 23 semanas.

La respuesta a la fertilización fue muy significativa en el $\mathrm{S}_{2}$, que incrementó la materia seca de los plantines un $70 \%$ desde la semana 13 a la 18 y $91 \%$ desde la 18 a la 23 , en tanto que para el caso del $\mathrm{S}_{1}$, los correspondientes incrementos fueron de $9 \%$ y $50 \%$ en los periodos indicados.

Para el caso de E. grandis, la incorporación de Trichoderma en los dos sustratos no tuvo incidencia de significación, ni antes ni después de la fertilización de la semana 16.

\section{Evaluación de Diferentes Dosis de Fertilización en la Producción de Plantines de Pinus taeda Creciendo en Sustrato Colonizado por Trichoderma harzianum}

Los resultados obtenidos para los diferentes parámetros evaluados en el del ensayo son resumidos en el Cuadro $\mathrm{N}^{\circ} 7$. 


\section{Cuadro $\mathrm{N}^{\circ} 7$}

RESULTADOS DEL ENSAYO

\begin{tabular}{|c|c|c|c|}
\hline Parámetros & $\begin{array}{c}\text { Diferencias significativas } \\
\text { con y sin Trichoderma } \\
\text { harzianum } \\
(p<0.05)\end{array}$ & $\begin{array}{l}\text { Diferencias significativas entre } \\
\text { tratamientos con Trichoderma } \\
\text { harzianum } \\
(P<0.05)\end{array}$ & Modelo ajustado a $p<0.05$ \\
\hline Altura & \multirow{8}{*}{ SI } & NO & 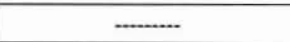 \\
\hline Diámetro & & $\mathrm{SI}$ & LINEAL \\
\hline Peso fresco total & & $\mathrm{SI}$ & CUADRÁTICO \\
\hline Peso fresco parte aérea & & $\mathrm{SI}$ & CUADRÁTICO \\
\hline Peso fresco radicular & & NO & (n).n. \\
\hline Peso seco total & & SI & LINEAL \\
\hline Peso seco parte aerea & & SI & CUADRATICO \\
\hline Peso seco radicular & & NO & ……n... \\
\hline$N(\%)$ & \multirow{4}{*}{ NO } & $\mathrm{SI}$ & LINEAL \\
\hline $\mathrm{P}(\%)$ Muestreo 1 & & NO & 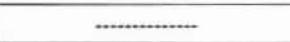 \\
\hline $\mathrm{P}(\%)$ Muestreo 2 & & NO & ................ \\
\hline $\mathrm{K}(\%)$ & & SI & LINEAL \\
\hline B ppm & SI & NO & ………... \\
\hline
\end{tabular}

Es posible observar que:

Hay diferencias estadisticamente significativas entre sustratos, siendo el Sustrato 1 (cáscara de pino compostado) el que presentó más altos recuentos de Trichoderma

La inoculación de sustrato previo a la siembra con Trichoderma harzianum tuvo efecto promotor en el crecimiento lo que se evidencia en todos los parámetros de calidad evaluados.

La dosis de fertilizante utilizada por la empresa conjuntamente con Trichoderma harzianum produjo mejor calidad de plántula que el tratamiento sin inocular.

Se desestima la incidencia de Botrytis cinerea en los plantines por ser muy baja su presencia.

Los plantines creciendo en sustrato colonizado por Trichoderma harzianum T1 a T4 presentan $15 \%$ más de diámetro que los plantines creciendo en sustrato sin inocular.

Los plantines creciendo en sustrato colonizado por Trichoderma harzianum presentan $24 \%$ más de altura que los plantines creciendo en sustrato sin inocular

Los plantines creciendo en sustrato colonizado por Trichoderma harzianum presentan 45 $\%$ más peso seco de parte aérea que los plantines creciendo en sustrato sin inocular. 
Los plantines creciendo en sustrato colonizado por Trichoderma harzianum presentan 25 $\%$ más peso seco parte radicular que los plantines creciendo en sustrato sin inocular.

Los plantines creciendo en sustrato colonizado por Trichoderma harzianum presentan $38 \%$ más peso seco total que los plantines creciendo en sustrato $\sin$ inocular.

Al comparar los tratamientos con diferentes dosis de $\mathrm{P}$ se aprecia que la concentración foliar es prácticamente constante.

Para el Boro se constata diferencias significativas entre el T0 y T1, lo que sugiere un efecto inmovilizador por parte de Trichoderma, aunque los valores de concentración foliar para todos los tratamientos son mayores que los rangos de referencia.

\section{CONCLUSIONES}

Existieron diferencias significativas entre sustratos en recuentos de sobrevivencia para unidades formadoras de colonia de Trichoderma.

La inoculación de Trichoderma harzianum tuvo efecto promotor en el crecimiento lo que queda evidenciado en parámetros de calidad evaluados en los plantines forestales obtenidos. incidencia.

Se desestimó la presencia de Botrytis cinerea en los plantines por ser muy baja su

\section{REFERENCIAS}

Batista de Oliveira, M., 2001. Regulamentacao para registro de biofungicidas no Brasil, Reuniao de controle biologico de fitopatogenos, Bento Gonzalves, RS. Anais, 2001.

Cabrera, R. y Tejera, R., 2002. Evaluación de diferentes dosis de fertilización en la Producción de plantines de Eucalyptus grandis creciendo en sustrato colonizado por Trichoderma harzianum. Montevideo. Facultad de Agronomia.Uruguay. $156 \mathrm{p}$.

Cardenas, D., 2003. Evaluación de calidad de plantines de Eucalyptus globulus ssp. globulus, E. grandis y Pino taeda con la incorporación de sustrato con Trichoderma harzianum. Montevideo. Facultad. de Agronomia. Uruguay $198 \mathrm{p}$.

Del Pino, A., 1997. Nutrición catiónica. Montevideo, Uruguay. Facultad de Agronomia 30 p.

James, R. and Beall, K., 1999. An evaluation of the effects of dazomet on soil- Bornediseases and conifer seedling production. USDA Forest Service, Luckey, Missoula, Montana.15p.

Lamondia, J.A. and Douglas, S.M., 1997. Sensitivity of Botrytis cinerea from Connecticut greenhouses to Benzimidazole and Dicarboximide fungicides. Plant Disease 81:729.732. 
Lucon, C., Nascimento, L. y Correa, C., 2001. Tratamento en post-coleita de mamao, Carica papaya, com isolados de Trichoderma sp. Anais de Reuniao de Controle Biologico de fitopatogenos, Bentos Goncalves, RS Brasil.

Reyna, R., 2000. Evaluación de métodos biológicos y químicos para el control de Botrytis cinerea en viveros de Eucalyptus globulus ssp.globulus. Montevideo. Facultad de Agronomia.Uruguay 70 p.

Romero, G, y Cardenas, D., 2001. Evaluacion de agente de biocontrol Trichodernma harzianum sobre Botrytis cinerea en producción de plantines de Eucalyptus grandis, E. globulus y Pinus taeda. VII Reuniao DE Controle Biologico de fitopatogenos, Bento Goncalves, RS, Brasil. 